\title{
Convalescent plasma anti-SARS-CoV-2 spike protein ectodomain and receptor-binding domain IgC correlate with virus neutralization
}

Eric Salazar, ${ }^{1,2}$ Suresh V. Kuchipudi, ${ }^{3,4}$ Paul A. Christensen, ${ }^{1}$ Todd Eagar, ${ }^{1,2}$ Xin Yi, ${ }^{1,2}$ Picheng Zhao, ${ }^{1}$ Zhicheng Jin, ${ }^{1}$ S. Wesley Long, ${ }^{1,2,5}$ Randall J. Olsen, ${ }^{1,2,5}$ Jian Chen, ${ }^{1,2}$ Brian Castillo, ${ }^{1,2}$ Christopher Leveque, ${ }^{1,2}$ Dalton Towers, ${ }^{6}$ Jason Lavinder, ${ }^{6}$ Jimmy Gollihar, ${ }^{7}$ Jose Cardona, ${ }^{7}$ Gregory Ippolito, ${ }^{6,8}$ Ruth Nissly, ${ }^{3}$ Ian Bird, ${ }^{3}$ Denver Greenawalt, ${ }^{3}$ Randall M. Rossi, ${ }^{9}$ Abhinay Gontu, ${ }^{3}$ Sreenidhi Srinivasan, ${ }^{9}$ Indira Poojary, ${ }^{9}$ Isabella M. Cattadori, ${ }^{4,5,10}$ Peter J. Hudson, ${ }^{4,9,10}$ Nicole M. Josleyn, ${ }^{11}$ Laura Prugar, ${ }^{11}$ Kathleen Huie, ${ }^{11}$ Andrew Herbert, ${ }^{11}$ David W. Bernard, ${ }^{1,2}$ John M. Dye, ${ }^{11}$ Vivek Kapur, ${ }^{4,9,12}$ and James M. Musser ${ }^{1,2,5}$

'Department of Pathology and Genomic Medicine, Houston Methodist Hospital, Houston, Texas, USA. ²Department of Pathology and Laboratory Medicine, Weill Cornell Medical College, New York, New York, USA. ${ }^{3}$ Penn State Animal Diagnostic Laboratory, Department of Veterinary and Biomedical Sciences, and ${ }^{4}$ Center for Infectious Disease Dynamics, Pennsylvania State University, University Park, Pennsylvania, USA. ${ }^{5}$ Center for Molecular and Translational Human Infectious Diseases, Houston Methodist Research Institute, Houston, Texas, USA. ${ }^{6}$ Department of Molecular Biosciences, University of Texas at Austin, Austin, Texas, USA. 'Combat Capabilities Development Command Army Research Laboratory - South, University of Texas, Austin, Texas, USA. ${ }^{8}$ Department of Oncology, Dell Medical School, University of Texas at Austin, Austin, Texas, USA. ${ }^{9}$ Huck Institutes of the Life Sciences and ${ }^{10}$ Department of Biology, Pennsylvania State University, University Park, Pennsylvania, USA. "US Army Medical Research Institute of Infectious Diseases, Frederick, Maryland, USA. ${ }^{2}$ Department of Animal Science, Pennsylvania State University, University Park, Pennsylvania, USA.

\begin{abstract}
The newly emerged severe acute respiratory syndrome coronavirus 2 (SARS-CoV-2) highlights the urgent need for assays that detect protective levels of neutralizing antibodies. We studied the relationship among anti-spike ectodomain (anti-ECD), antireceptor-binding domain (anti-RBD) IgC titers, and SARS-CoV-2 virus neutralization (VN) titers generated by 2 in vitro assays using convalescent plasma samples from 68 patients with COVID-19. We report a strong positive correlation between both plasma anti-RBD and anti-ECD IgC titers and in vitro VN titers. The probability of a VN titer of $\geq 160$, the FDA-recommended level for convalescent plasma used for COVID-19 treatment, was $\geq 80 \%$ when anti-RBD or anti-ECD titers were $\geq 1: 1350$. Of all donors, $37 \%$ lacked VN titers of $\geq 160$. Dyspnea, hospitalization, and disease severity were significantly associated with higher VN titer. Frequent donation of convalescent plasma did not significantly decrease VN or IgG titers. Analysis of 2814 asymptomatic adults found 73 individuals with anti-ECD IgG titers of $\geq 1: 50$ and strong positive correlation with anti-RBD and VN titers. Fourteen of these individuals had VN titers of $\geq 1: 160$, and all of them had anti-RBD titers of $\geq 1: 1350$. We conclude that anti-RBD or anti-ECD IgG titers can serve as a surrogate for VN titers to identify suitable plasma donors. Plasma anti-RBD or anti-ECD titers of $\geq 1: 1350$ may provide critical information about protection against COVID-19 disease.
\end{abstract}

\section{Introduction}

The recently emerged severe acute respiratory syndrome coronavirus 2 (SARS-CoV-2), a novel coronavirus that causes coronavirus disease 2019 (COVID-19) disease, has spread globally and is now responsible for massive human morbidity and mortality. The pathogen was first documented to cause severe respiratory infections in humans in Wuhan, China, beginning in late December 2019 (1). Soon thereafter, the SARS-CoV-2 virus was characterized as a member of the Betacoronavirus genus and recognized to be related to several bat coronaviruses, SARS, and Middle East Respiratory Syndrome (MERS) coronaviruses. SARS-CoV-2 spread was unusually rapid, and COVID-19 disease has now been reported in virtually all major population centers globally. In the US, more than

Conflict of interest: The authors have declared that no conflict of interest exists. Copyright: () 2020, American Society for Clinical Investigation.

Submitted: June 9, 2020; Accepted: September 2, 2020; Published: November 16, 2020 Reference information: J Clin Invest. 2020;130(12):6728-6738.

https://doi.org/10.1172/JCl141206.
1,500,000 COVID-19 cases have been documented and the virus has caused greater than 100,000 deaths nationwide. Many metropolitan regions have been especially affected, including, but not limited to Seattle, New York City, Chicago, Miami, and Detroit (2).

Management of COVID-19 infection has predominantly involved aggressive support care. Various treatment approaches are being studied, including direct viral replication inhibition (3), antiinflammatory drugs, and passive antibody therapies. Currently, the only available passive antibody therapy for patients with COVID-19 is transfusion of convalescent plasma obtained from recovered patients. The therapy is safe, and multiple emerging lines of evidence, including historical precedent, preclinical animal studies, small case series, and matched observational studies, suggest that convalescent plasma is efficacious in the treatment of COVID-19 (4, 5). Clinical trials assessing efficacy in specific patient populations are underway, and clinical trials assessing the use of hyperimmune IgG may begin soon (6).

The FDA has recommended (7) that convalescent plasma with a virus-neutralizing $(\mathrm{VN})$ antibody titer of $\geq 1: 160$ be used for ther- 
apeutic transfusion. While VN assays with live virus are considered the gold standard, these are not widely available, in part because they are labor intensive to run, cumbersome, and require a biosafety level 3 laboratory. Assays with pseudotype virus offer considerable advantages over live virus assays. These are safe to use in a biosafety level 2 environment and have the potential to assay responses to specific target viral proteins; however, cell culture and maintenance of suitable cell lines is still required. Thus, these assays are not readily integrated into the available donor testing infrastructure. Inasmuch as the VN titers in most donor plasma are not known before transfusion, a more facile method to identify suitable convalescent plasma donors is needed. This is an especially pressing matter, as an increasing number of patients with COVID-19 are being treated globally with convalescent plasma. For example, under an FDAapproved expanded access protocol, greater than 50,000 transfusions have already occurred in the US (8).

The trimeric spike (S) protein made by SARS-CoV-2 is a large molecule that is critical to virus dissemination and pathogenesis. $S$ protein is a densely glycosylated molecule present on the surface of the virus. S protein mediates binding of the SARS-CoV-2 virus to the host angiotensin-converting enzyme 2 (ACE2) receptors, thereby acting as the first step in cell entry and infection. Recent work has shown that SARS-CoV-2 and SARS-CoV-1 share the same ACE2 receptor. The molecular mechanism used by $S$ protein to gain entry into host cells is complex and involves a region of the molecule known as the receptor-binding domain (RBD). Engagement of S protein with the host receptor results in considerable changes in molecular conformation. The $\mathrm{S}$ protein has a critical function in host-cell entry and, thus, is a major target for vaccine research and antibody-mediated VN efforts.

Many lines of evidence from studies of SARS-CoV-1, MERS, and SARS-CoV-2 show that infected hosts make antibodies directed against $S$ protein (9-16). In addition, immunization with $S$ protein can protect laboratory animals against experimental infection with SARS-CoV-1, MERS-CoV, and SARS-CoV-2 (17-21). Similarly, IgG directed against $S$ protein has been reported to have in vitro $\mathrm{VN}$ activity.

The goal of this study was to test the hypothesis that anti-S ectodomain (anti-ECD) and/or anti-RBD IgG titers are correlated with VN titers and, thus, could be used as a surrogate marker to identify plasma donors with titers above the FDA threshold value of 1:160. To test this hypothesis, we studied plasma and serum samples from 68 recovered patients with COVID-19 with documented disease based on a positive molecular test for SARS-CoV-2. VN titer was determined independently in 2 laboratories using 2 different in vitro assays. The results show a strong positive correlation between anti-RBD and anti-ECD plasma IgG ELISA titers and the magnitude of in vitro VN. Specifically, we report that there is an $80 \%$ probability or greater of a VN titer at or above the FDA-recommended level of 1:160 for COVID-19 convalescent plasma with anti-RBD or anti-ECD IgG titers of $\geq 1: 1350$. The results provide an important quantitative target for therapeutic and prophylactic treatments. We also found that convalescent donors maintain high-titer antiRBD and anti-ECD IgG with in vitro VN activity over many weeks. Frequent plasma donations do not cause a significant decrease in antibody or VN titers. Finally, analysis of anti-ECD and anti-RBD IgG titers in 2814 asymptomatic individuals in a surveillance cohort identified 14 individuals with VN titers of $\geq 1: 160$, with all of these having an anti-RBD titer of $\geq 1: 1350$. Thus, some asymptomatic individuals may have plasma suitable for therapeutic use and may have a degree of relative immunity against SARS-CoV-2.

\section{Results}

Plasma donor characteristics. Ninety-three samples from sixty-eight unique COVID-19 convalescent plasma donors were assessed (Tables 1 and 2). The average age was 45 years (range 23-78 years), and 36 of the donors were female. Most donors had severity scores (as defined in the Methods) consistent with mild-to-moderate disease, with $44 \%$ (30 of 68) having a symptom severity score of $1 ; 32 \%$ (22 of 68 ) having score of $2 ; 10 \%$ (7 of 68 ) having a score of $3 ; 7 \%$ ( 5 of 68 ) having a score of 4 ; and $6 \%$ (4 of 68) having a score of 5 . Sixteen donors required hospitalization, with an average length of stay of 4 days (range 2-13 days). Thirteen individuals donated more than once (range 1-7 times), with most (9 of 13) donating twice only. For all samples assessed, the median interval from symptom onset to donation visit was 32 days (range, 17-53 days; IQR, 28-36 days), and the median interval from symptom resolution to donation visit was 20 days (range, 15-38 days; IQR, 17-25 days).

We also studied plasma from 73 asymptomatic individuals identified during an institutional surveillance program involving 2814 individuals (22). Of these 2814 individuals, 73 had anti-ECD ELISA titers of $\geq 50$. The average age of these 73 asymptomatic individuals was 38 years (range, 20-69 years), and 56 of the 73 individuals (77\%) were female (Supplemental Table 1; supplemental material available online with this article; https://doi. org/10.1172/JCI141206DS1).

$V N$ titers in convalescent plasma donors. VN titers in samples from COVID-19 convalescent plasma donors were assessed with a traditional microneutralization assay evaluating protection from virus infection, as determined by crystal violet staining 3 days after infection. Plasma samples from the majority of donors (43 of $68 ; 63 \%$ ) had a VN titer of $\geq 1: 160$, the FDA-recommended VN antibody titer for convalescent plasma to be used for therapeutic transfusion purposes. In contrast, 25 of 68 donors (37\%) had a plasma titer below this recommended cut-off value (Figure $1 \mathrm{~A}$ and Tables 1 and 2).

Correlation between 2 VN assays. VN titers were assessed blinded (that is, without knowledge of the data generated by laboratory one) in a second laboratory with a different microneutralization assay (VN2) that determined the percentage of infected cells 24 hours after infection using a SARS-CoV-2-specific monoclonal antibody and a fluorescently labeled secondary antibody. The results from the $2 \mathrm{VN}$ assays were highly correlated $(r=0.66, P<$ 0.001) (Figure 1, B and C).

Association between ELISA IgG titers and VN titer. Recognizing the urgent need for assays that could serve as a surrogate for VN, we assessed the association between ELISA antiECD and anti-RBD IgG titers and VN titers. The results of all 4 assays (anti-ECD and anti-RBD ELISAs, VN, and VN2) were strongly correlated (Figure 1C). Anti-RBD IgG had a numerically, but not statistically, greater correlation compared with 
Table 1. Demographics and characteristics of convalescent plasma donors and samples

Sample Subject ID Sex Age Severity Hospitalization LOS Symptom Days after Days after Visit $\quad$ ECD $\quad$ RBD $\quad$ VN $\quad$ VN2 Clade (yr)

(d) symptom symptom resolution

\begin{tabular}{|c|c|c|c|c|c|c|c|c|c|c|c|c|c|c|c|}
\hline 1 & НМНООО1 & $M$ & 44 & 1 & NO & $\mathrm{N} / \mathrm{A}$ & 3 & 20 & 17 & 1 & 150 & 1350 & 320 & 53.2 & $\mathrm{~A} 2 \mathrm{a}$ \\
\hline 2 & НМНООО1 & M & 44 & 1 & NO & $\mathrm{N} / \mathrm{A}$ & 3 & 24 & 21 & 2 & $\mathrm{~N} / \mathrm{A}$ & $\mathrm{N} / \mathrm{A}$ & 640 & 48.6 & $\mathrm{~A} 2 \mathrm{a}$ \\
\hline 3 & НМНООО1 & M & 44 & 1 & NO & $\mathrm{N} / \mathrm{A}$ & 3 & 27 & 24 & 3 & 150 & 450 & 320 & 53.3 & $\mathrm{~A} 2 \mathrm{a}$ \\
\hline 4 & НМНООО1 & M & 44 & 1 & NO & $\mathrm{N} / \mathrm{A}$ & 3 & 31 & 28 & 4 & 150 & 800 & 320 & 46.8 & $\mathrm{~A} 2 \mathrm{a}$ \\
\hline 5 & НМНООО1 & M & 44 & 1 & NO & $\mathrm{N} / \mathrm{A}$ & 3 & 34 & 31 & 5 & 150 & 800 & 320 & 96.3 & $\mathrm{~A} 2 \mathrm{a}$ \\
\hline 6 & НМНООО1 & M & 44 & 1 & NO & $\mathrm{N} / \mathrm{A}$ & 3 & 38 & 35 & 6 & 450 & 450 & 160 & 69.4 & $\mathrm{~A} 2 \mathrm{a}$ \\
\hline 7 & НМНООО1 & M & 44 & 1 & NO & $\mathrm{N} / \mathrm{A}$ & 3 & 41 & 38 & 7 & 450 & 450 & 80 & 71.5 & $\mathrm{~A} 2 \mathrm{a}$ \\
\hline 8 & HМHOOO2 & M & 54 & 1 & NO & $\mathrm{N} / \mathrm{A}$ & 13 & 28 & 15 & 1 & 50 & 150 & 40 & 1.5 & $\mathrm{~A} 2 \mathrm{a}$ \\
\hline 9 & НМHOООЗ & $M$ & 36 & 1 & NO & $\mathrm{N} / \mathrm{A}$ & 7 & 25 & 18 & 1 & 450 & 1350 & 80 & 38.4 & B \\
\hline 10 & НМНОООЗ & M & 36 & 1 & NO & $\mathrm{N} / \mathrm{A}$ & 7 & 28 & 21 & 2 & 150 & 450 & 80 & 10.4 & B \\
\hline 11 & НМНОООЗ & M & 36 & 1 & NO & $\mathrm{N} / \mathrm{A}$ & 7 & 33 & 26 & 3 & 450 & 800 & 80 & 7.6 & B \\
\hline 12 & НМНОООЗ & M & 36 & 1 & NO & $\mathrm{N} / \mathrm{A}$ & 7 & 35 & 28 & 4 & 450 & 450 & 0 & 8 & B \\
\hline 13 & НМНОООЗ & $M$ & 36 & 1 & NO & $\mathrm{N} / \mathrm{A}$ & 7 & 40 & 33 & 5 & 450 & 450 & 320 & 19.8 & B \\
\hline 14 & НМНОООЗ & M & 36 & 1 & NO & $\mathrm{N} / \mathrm{A}$ & 7 & 42 & 35 & 6 & 150 & 450 & 20 & 1.6 & B \\
\hline 15 & HМHOOO4 & $\mathrm{F}$ & 54 & 2 & NO & $\mathrm{N} / \mathrm{A}$ & 18 & 32 & 14 & 1 & 1350 & 1350 & 320 & 270.7 & $\mathrm{~N} / \mathrm{A}$ \\
\hline 16 & HМHOOO4 & $\mathrm{F}$ & 54 & 2 & NO & $\mathrm{N} / \mathrm{A}$ & 18 & 36 & 18 & 2 & 1350 & 1350 & 640 & 128.5 & $\mathrm{~N} / \mathrm{A}$ \\
\hline 17 & HМHOOO9 & $\mathrm{F}$ & 38 & 2 & NO & $\mathrm{N} / \mathrm{A}$ & 12 & 30 & 18 & 1 & 450 & 450 & 80 & 4.8 & N/A \\
\hline 18 & НМН0011 & $\mathrm{F}$ & 67 & 1 & NO & $\mathrm{N} / \mathrm{A}$ & 11 & 28 & 17 & 1 & 0 & 50 & 40 & 8 & $\mathrm{~A} 2 \mathrm{a}$ \\
\hline 19 & HМH0012 & $F$ & 46 & 1 & NO & $\mathrm{N} / \mathrm{A}$ & 16 & 30 & 14 & 1 & 150 & 450 & 320 & 30 & N/A \\
\hline 20 & НМН0013 & $\mathrm{F}$ & 43 & 1 & NO & $\mathrm{N} / \mathrm{A}$ & 11 & 28 & 17 & 1 & 1350 & 3200 & 320 & 214.2 & $\mathrm{~A} 2 \mathrm{a}$ \\
\hline 21 & НМН0016 & $\mathrm{F}$ & 47 & 1 & NO & $\mathrm{N} / \mathrm{A}$ & 13 & 32 & 19 & 1 & 4050 & 4050 & 320 & 234.2 & $\mathrm{~A} 2 \mathrm{a}$ \\
\hline 22 & НМН0020 & $\mathrm{F}$ & 41 & 2 & NO & $\mathrm{N} / \mathrm{A}$ & 2 & 17 & 15 & 1 & 50 & 200 & 20 & $\mathrm{~N} / \mathrm{A}$ & $\mathrm{N} / \mathrm{A}$ \\
\hline 23 & HМH0O28 & M & 23 & 1 & NO & $\mathrm{N} / \mathrm{A}$ & 12 & 31 & 19 & 1 & 150 & 150 & 20 & 0.6 & $\mathrm{~A} 2 \mathrm{a}$ \\
\hline 24 & НМНОО29 & $\mathrm{F}$ & 66 & 1 & NO & $\mathrm{N} / \mathrm{A}$ & 6 & 22 & 16 & 1 & 150 & 450 & 80 & 9.3 & $\mathrm{~A} 2 \mathrm{a}$ \\
\hline 25 & НМН0032 & M & 65 & 2 & NO & $\mathrm{N} / \mathrm{A}$ & 11 & 25 & 14 & 1 & 450 & 4050 & 320 & 94.8 & $\mathrm{~N} / \mathrm{A}$ \\
\hline 26 & HМH0035 & M & 50 & 2 & NO & $\mathrm{N} / \mathrm{A}$ & 14 & 28 & 14 & 1 & 1350 & 3200 & 320 & 47.3 & B \\
\hline 27 & НМН0035 & M & 50 & 2 & NO & $\mathrm{N} / \mathrm{A}$ & 14 & 38 & 24 & 2 & 1350 & 1350 & 640 & 72 & B \\
\hline 28 & НМНОО40 & M & 52 & 2 & NO & $\mathrm{N} / \mathrm{A}$ & 12 & 29 & 17 & 1 & $\mathrm{~N} / \mathrm{A}$ & $\mathrm{N} / \mathrm{A}$ & 1280 & 417 & $\mathrm{~A} 2 \mathrm{a}$ \\
\hline 29 & НМН0040 & M & 52 & 2 & NO & $\mathrm{N} / \mathrm{A}$ & 12 & 35 & 23 & 2 & 4050 & 4050 & 640 & 724.5 & $\mathrm{~A} 2 \mathrm{a}$ \\
\hline 30 & НМНОО40 & M & 52 & 2 & NO & $\mathrm{N} / \mathrm{A}$ & 12 & 37 & 25 & 3 & 1350 & 4050 & 320 & 274 & $\mathrm{~A} 2 \mathrm{a}$ \\
\hline 31 & HМH0045 & $\mathrm{F}$ & 23 & 1 & NO & $N / A$ & 9 & 33 & 24 & 1 & 4050 & 1350 & 1280 & 63.8 & $\mathrm{~A} 2 \mathrm{a}$ \\
\hline 32 & НМН0049 & $\mathrm{F}$ & 57 & 1 & NO & $\mathrm{N} / \mathrm{A}$ & 12 & 27 & 15 & 1 & 150 & 450 & 320 & 47 & $\mathrm{~N} / \mathrm{A}$ \\
\hline 33 & HМHO050 & M & 41 & 2 & NO & $\mathrm{N} / \mathrm{A}$ & 7 & 30 & 23 & 1 & 1350 & 1350 & 320 & 18.9 & N/A \\
\hline 34 & HМHO050 & M & 41 & 2 & NO & $\mathrm{N} / \mathrm{A}$ & 7 & 33 & 26 & 2 & 150 & 150 & 320 & 21.4 & N/A \\
\hline 35 & НМH0051 & $\mathrm{F}$ & 50 & 1 & NO & $\mathrm{N} / \mathrm{A}$ & 16 & 30 & 14 & 1 & 150 & 450 & 160 & 58.4 & N/A \\
\hline 36 & HМH0052 & $\mathrm{F}$ & 27 & 3 & YES & 2 & 17 & 31 & 14 & 1 & 1350 & 1350 & 160 & 100.1 & $\mathrm{~A} 2 \mathrm{a}$ \\
\hline 37 & НМН0053 & M & 29 & 2 & NO & $\mathrm{N} / \mathrm{A}$ & 10 & 28 & 18 & 1 & 450 & 450 & 160 & 18.9 & $\mathrm{~N} / \mathrm{A}$ \\
\hline 38 & HМH0055 & M & 61 & 3 & YES & 3 & 14 & 33 & 19 & 1 & 1350 & 3200 & 320 & 134.1 & B \\
\hline 39 & HМH0057 & $\mathrm{F}$ & 44 & 2 & NO & $\mathrm{N} / \mathrm{A}$ & 6 & 34 & 28 & 1 & 450 & 450 & 160 & 111.5 & $\mathrm{~A} 2 \mathrm{a}$ \\
\hline 40 & НМH0O62 & $\mathrm{F}$ & 24 & 1 & NO & $\mathrm{N} / \mathrm{A}$ & 13 & 32 & 19 & 1 & 1350 & 1350 & 10 & 443.9 & $\mathrm{~A} 2 \mathrm{a}$ \\
\hline 41 & НМH0О62 & $\mathrm{F}$ & 24 & 1 & NO & $\mathrm{N} / \mathrm{A}$ & 13 & 35 & 22 & 2 & 1350 & 1350 & 1280 & 288.8 & $\mathrm{~A} 2 \mathrm{a}$ \\
\hline 42 & НМН0069 & $\mathrm{F}$ & 49 & 1 & NO & $\mathrm{N} / \mathrm{A}$ & 6 & 28 & 22 & 1 & 450 & 450 & 80 & NA & $\mathrm{A} 2 \mathrm{a}$ \\
\hline 43 & НМНОО69 & $\mathrm{F}$ & 49 & 1 & NO & $\mathrm{N} / \mathrm{A}$ & 6 & 32 & 26 & 2 & 450 & 450 & 80 & 35.3 & $\mathrm{~A} 2 \mathrm{a}$ \\
\hline 44 & НМНОО7О & $\mathrm{F}$ & 37 & 2 & NO & $\mathrm{N} / \mathrm{A}$ & 19 & 38 & 19 & 1 & 450 & 1350 & 160 & 126.7 & $\mathrm{~A} 2 \mathrm{a}$ \\
\hline 45 & НМН0072 & $\mathrm{F}$ & 23 & 1 & NO & $\mathrm{N} / \mathrm{A}$ & 13 & 29 & 16 & 1 & 0 & 0 & 0 & $\mathrm{~N} / \mathrm{A}$ & $\mathrm{N} / \mathrm{A}$ \\
\hline 46 & НМH0072 & $\mathrm{F}$ & 23 & 1 & NO & $\mathrm{N} / \mathrm{A}$ & 13 & 37 & 24 & 2 & 50 & 50 & 0 & $\mathrm{~N} / \mathrm{A}$ & $\mathrm{N} / \mathrm{A}$ \\
\hline 47 & НМН0088 & $\mathrm{F}$ & 29 & 1 & NO & $\mathrm{N} / \mathrm{A}$ & 3 & 21 & 18 & 1 & 0 & 50 & 20 & $\mathrm{~N} / \mathrm{A}$ & $\mathrm{A} 2 \mathrm{a}$ \\
\hline 48 & HМH0089 & $\mathrm{F}$ & 42 & 2 & NO & $\mathrm{N} / \mathrm{A}$ & 18 & 38 & 20 & 1 & 450 & 450 & 160 & 95 & $\mathrm{~A} 2 \mathrm{a}$ \\
\hline 49 & НМНОО9О & $M$ & 33 & 1 & NO & $\mathrm{N} / \mathrm{A}$ & 3 & 37 & 34 & 1 & 150 & 150 & 1280 & 51.3 & $\mathrm{~A} 2 \mathrm{a}$ \\
\hline
\end{tabular}

F, female; M, male; LOS, length of stay; ECD, anti-spike ectodomain; RBD, receptor-binding domain; VN, virus neutralization. In the LOS column, N/A denotes not available because the donor was not hospitalized. 
Table 2. Demographics and characteristics of convalescent plasma donors and samples

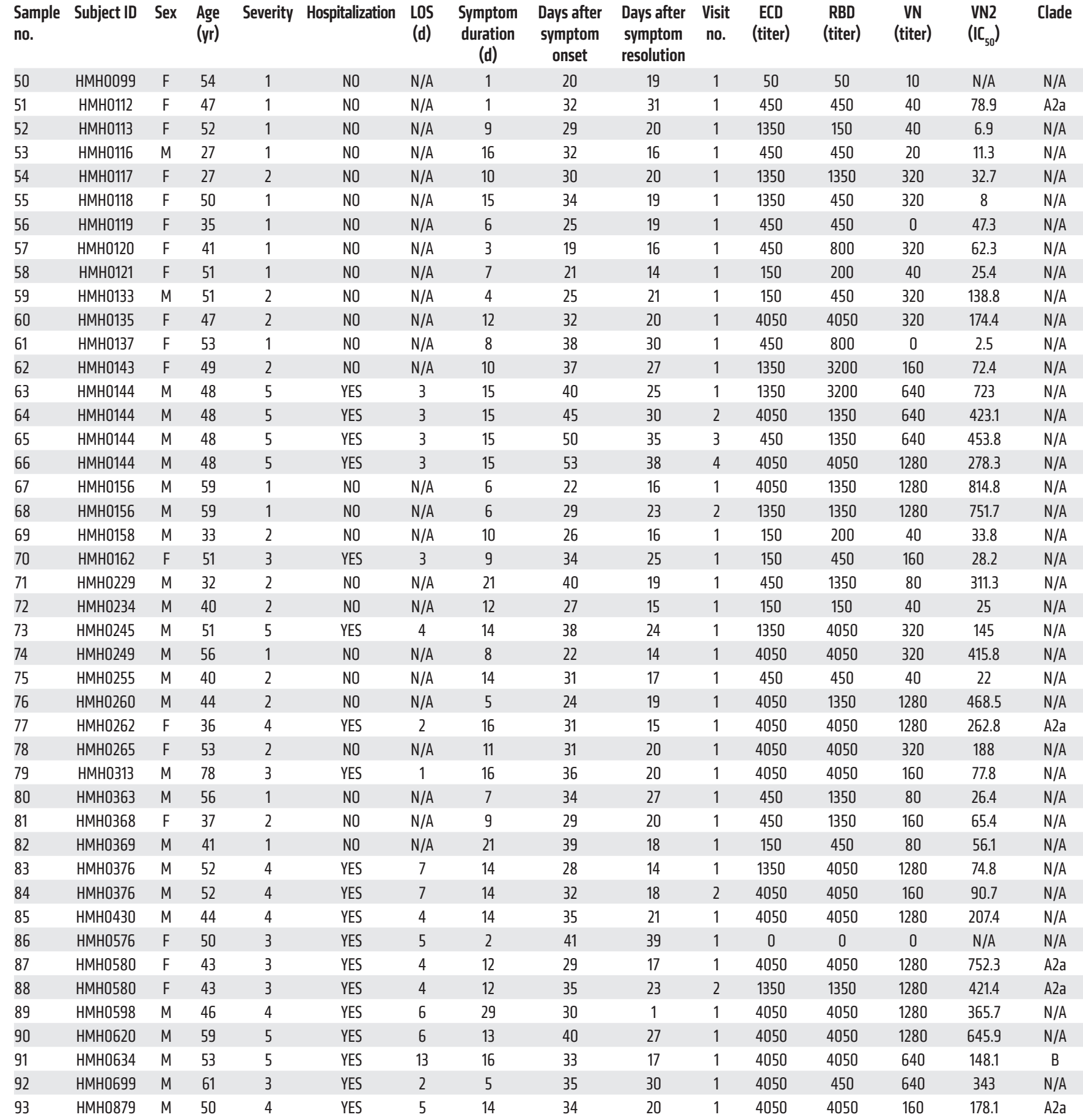

F, female; M, male; LOS, length of stay; ECD, anti-spike ectodomain; RBD, receptor-binding domain; VN, virus neutralization. ; VN, virus neutralization. In the LOS column, N/A denotes not available because the donor was not hospitalized.

anti-ECD (0.67 versus 0.62$)$ with both microneutralization assays. We found that more than $80 \%$ of donors had a VN titer of $\geq 1: 160$ in convalescent plasma when their serum anti-RBD or anti-ECD titers were 1:1350 or higher (Figure 2). Among 61 samples from plasma donors with a VN titer of $\geq 1: 160,59$ had an anti-RBD assessment, and 41 (70\%) had an anti-RBD titer of $\geq 1: 1350$. Conversely, only 4 of 45 samples with an anti-RBD titer of $\geq 1: 1350$ had a $\mathrm{VN}$ of $\leq 160$, indicating a positive predictive value for $V N$ titers of $\geq 1: 160$ of $91 \%$. Importantly, samples from naive human plasma specimens obtained before the discovery of SARS-CoV-2 had no detectable titer in any of the 4 assays (data not shown). 

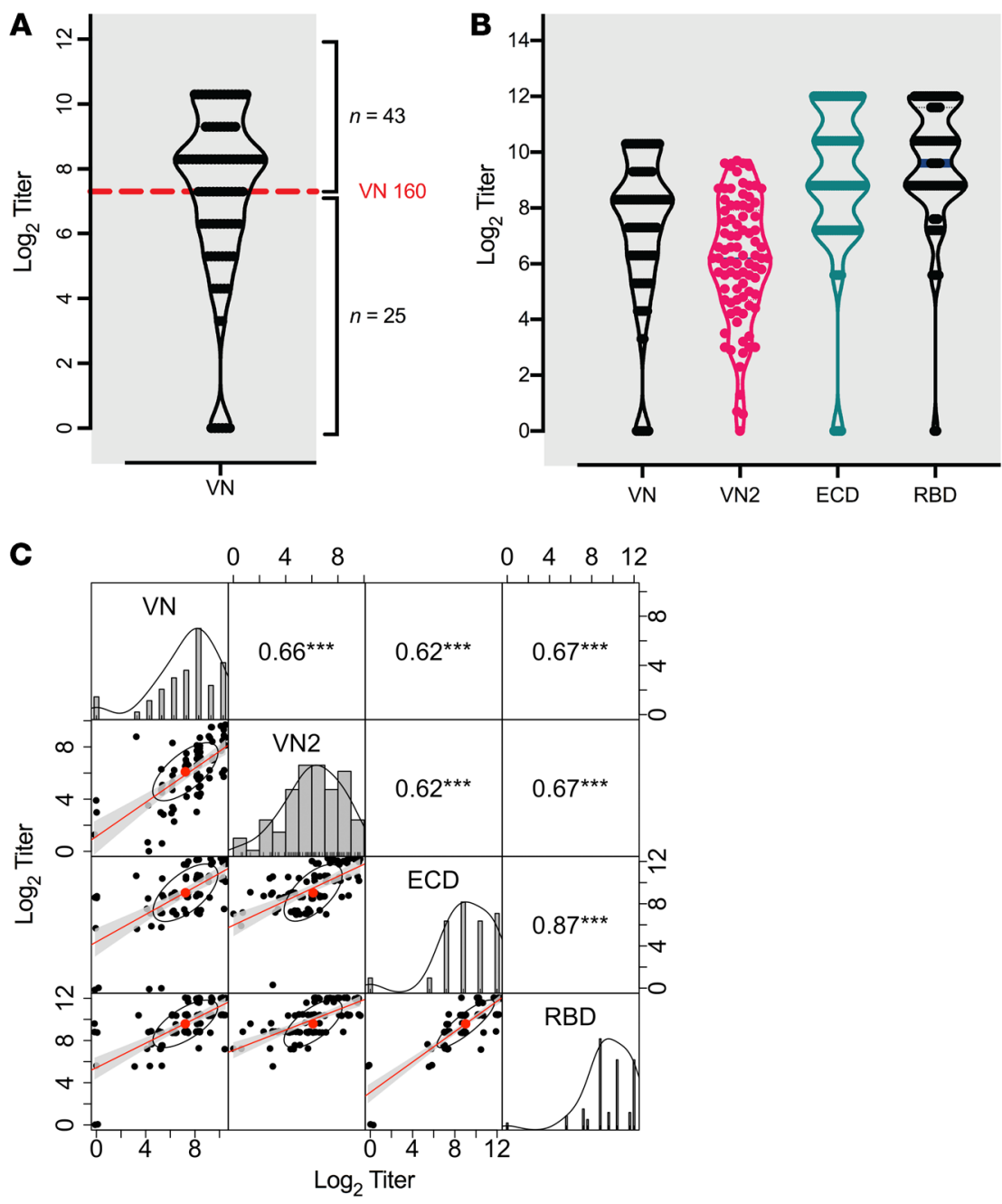

Relationship between antibody titers and donor characteristics. As approximately one-third of donors lacked convalescent plasma with the FDA-recommended VN titer cut-off of $\geq 1: 160$, we sought to identify donor characteristics that may be associated with a higher IgG titer. Such characteristics could aid donor recruitment efforts by identifying the recovered patients who may have mounted a strong humoral response. We found that the presence of dyspnea during COVID-19 disease, hospitalization requirement, and more severe disease were all positively and significantly associated with higher IgG titers in all assays (Figure 3). Duration of disease symptoms was not associated with titer. Nor was there an association with time of plasma collection since symptom onset and titer in the donor population. All collections occurred more than 14 days after symptom resolution (as required by the FDA). These results suggest that donors had already plateaued in their IgG titer at the time plasma was obtained, as there was no appreciable trend in titer increase over time (Supplemental Figure $1, \mathrm{~A}$ and $\mathrm{B})$. There was a trend toward lower titers in younger age group donors and in female donors, but these findings were not consistent across all assays (Supplemental Figure 1, C and D).

$V N$ titers over time from the same convalescent plasma donors. Thirteen individuals donated convalescent plasma more than once (range, 2-7 donations). The availability of longitudinal sam-
Figure 1. Patterns of VN and ELISA titers. (A) Violin plot of distribution of $\mathrm{VN}$ titers at initial donation. The number of donor cases above $(n=43)$ and below $(n=25)$ the $\mathrm{VN} \geq 160$ cut-off value is reported. (B) Violin plots showing similar patterns of distribution of titers at initial donation for the $2 \mathrm{VN}$ assays, together with the reciprocal ELISA IgC titers for plasma anti-ECD protein (ECD) and anti-RBD IgC (RBD). (C) Pair-wise Pearson correlations showing the correlation coefficient $(r)$ and related significance value $\left.{ }^{* * *} P<0.001\right)$ above the diagonal as well as the bivariate scatter plots (jittered points represented as black dots) with linear regression fit (red line), Cls (gray shading), correlation value (red points), and correlation ellipse (black ellipses) below the diagonal. The density plot (black line) and histogram of each variable is reported along the diagonal. Data are presented in $\log _{2}$ scale of reciprocal titers for $\mathrm{VN}$, anti-ECD IgG, and anti-RBD IgC and in $\mathrm{IC}_{50}$ units for VN2. The sample sizes for which the correlation coefficients were derived are as follows: VN-VN2, 86; VN-ECD, 91; VN-RBD, 91; VN2-ECD, 84; VN2-RBD, 84 ECD-RBD, 91 ples from the same plasma donors permitted us to assess the arc of anti-ECD and anti-RBD IgG titers and VN over time within individuals. There was no significant decrease in IgG titers, as assessed by the ELISA or VN titer (Supplemental Figure 2), even among donors who donated twice per week for up to 7 donations. Thus, we observed stable, high titers both within and between individual donors.

Relationship between infecting strain clade and VN titer. We had available the virus genome sequences obtained from clinical samples (e.g., nasopharyngeal swab, oropharyngeal swab, or sputum) from 25 plasma donors. Eighty-four percent (21 of 25) of donors had been infected with strain A2a, and the remaining donors had been infected with strain B. Although the number of specimens was small, we tested the hypothesis that a relationship exists between the VN titer and genetic clade of the infecting SARSCoV-2 strain. No definitive relationship was evident from analysis of the available data (Table 1).

Asymptomatic individuals and VN titers. Having established a relationship between IgG titer and in vitro SARS-CoV-2 VN titer, we next determined IgG titers in a sample of 2814 asymptomatic adults screened under a surveillance protocol. We found that 73 of 2814 (2.5\%) individuals had anti-ECD and anti-RBD IgG ELISA titers of $\geq 1: 50$, of which 27 had anti-RBD or anti-ECD IgG titers of $\geq 1: 1350$ 


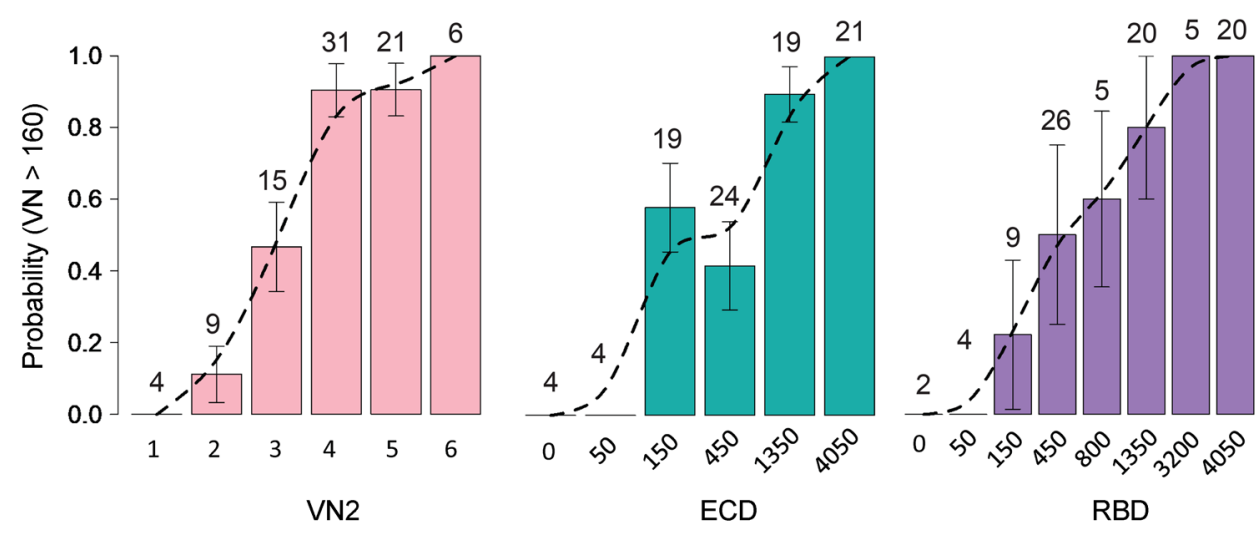

Figure 2. Prevalence of donors with VN $>\mathbf{1 6 0}$ for VN2, ECD, and RBD. Probabilities of VN $\geq 160$ were plotted for 6 range classes, with an interclass interval of $1.8 \log _{2} \mathrm{IC}_{50}$ values (class 1, <2; class 2, 2-12; class 3, 12-42; class 4, 42-147; class 5, 147-512; and class 6, >512) or observed classes for ECD ( $n=6$ ) and RBD $(n=8)$ reciprocal ELISA titers. A spline curve (dotted line, smoothness shape $=1$ ) has been fitted to the probability values and standard errors $($ bars) are reported. The numbers of donor samples are shown above the bars.

(Supplemental Table 1). Among the 73 specimens from asymptomatic individuals analyzed for $\mathrm{VN}$ titer, the correlation among antiECD, anti-RBD, and VN titer remained highly significant, with $P<0.001$ in all comparisons (Supplemental Figure 3). In all cases in which VN titer was $\geq 1: 160$, the anti-RBD titer was $\geq 1: 1350$.

\section{Discussion}

In the absence of an efficacious vaccine to prevent COVID-19 disease, there is a pressing need for assays that detect neutralizing antibodies against SARS-CoV-2. Here, we studied the relationship between anti-RBD and anti-ECD IgG titers present in convalescent plasma obtained from patients with COVID-19 and in vitro SARSCoV-2 VN. We discovered a strong positive association between anti-RBD and anti-ECD plasma IgG titers and in vitro VN titer.

The data provide important evidence that anti-ECD and antiRBD IgG titers are a suitable proxy for VN titer. Given the limited availability of VN assays, which are technically complex; require days to set up, run, and interpret; and need a biosafety level 3 laboratory when performed with live native SARS-CoV-2 virus, and the relative ease with which ELISAs can be implemented and performed in a high-throughput fashion, we believe our data provide a guidepost for proxy assessments of $\mathrm{VN}$ titers relevant to the COVID-19 pandemic.

We found that although both anti-ECD and anti-RBD IgG titers correlate well and significantly with in vitro VN, anti-RBD IgG titer had a tendency for a stronger correlation than anti-ECD IgG titer. This finding is consistent with a study showing clustering of VN epitopes in the SARS-CoV-1 RBD domain (23). In this study, neutralizing monoclonal antibodies mapped to a region of $\mathrm{RBD}$ that has a critical role in attachment to the host ACE2 receptor. Given that the RBD is also the important region for ACE2 receptor binding for SARS-CoV-2 $(24,25)$, it is not surprising that anti-RBD IgG titers correlate well with VN titers. Importantly, our data from convalescent plasma donors show that anti-RBD or anti-ECD IgG titers of 1:1350 discriminated the presence of an adequate VN titer, as recommended by the FDA for COVID-19 convalescent plasma, with a probability of approximately $80 \%$. Using this anti-RBD or anti-ECD IgG titer cut-off, a proportion of donors and plasma units with adequate VN titer would be excluded from use. In an effort to rapidly identify donors and plasma units likely to have efficacy, this exclusion rate is acceptable, while the alternative transfusion of patients with convalescent plasma units with low or no titer of anti-SARS-CoV-2 antibody, is not. Future studies are required to determine if a VN titer of $\geq 1$ :160 has therapeutic benefit. Regardless, our findings clearly indicate that an anti-RBD IgG titer cut-off can be established that serves as a suitable proxy for $\mathrm{VN}$ titer.

Our findings greatly expand on recent work showing a relationship between anti-S ELISA and microneutralization titer in 9 samples using a microneutralization assay 48 hours after infection to assess "whole-well" optical density (13). Suthar et al. have also demonstrated that RBD-specific IgG endpoint titer correlates well with a focus-reduction neutralization assay (12). Li et al. reported a positive correlation between SARS-CoV-2 VN titer and S-RBDspecific IgG titer, with a serum VN titer of 1:80 as approximately equivalent to a titer of 1:1280 for S-RBD-specific IgG (26). Because of differences in the $\mathrm{VN}$ assay used, their titers and those we report here are not equivalent (27). Harvala et al. also reported that $\mathrm{VN}$ and anti-S ELISA titers were correlated (28), although there were several differences between that study and ours. For example, all donors were male, plasma was collected $>28$ days after symptom resolution, a different virus strain was used, no repeat donors were studied, and the association with clinical symptoms was not assessed. In addition, they did not study samples obtained during community screening of asymptomatic individuals. Herein, we compared results from 2 independent neutralization assays run blinded in 2 independent laboratories. The traditional VN assay assessed protection from SARS-CoV-2 virus infection, as determined by the presence of cytopathic effect 3 days after infection. In contrast, VN2 analyzed the percentage of SARS-CoV-2 virus-infected cells 24 hours after infection as a measure of early virus replication and susceptibility to host-cell infection. These 2 different approaches to VN assessment, and the robustness of the correlation between the results of the 2 different assays, add confidence to our conclusion that anti-RBD IgG and anti-ECD IgG titers measured by ELISA serve as a very reliable surrogate of $\mathrm{VN}$. 

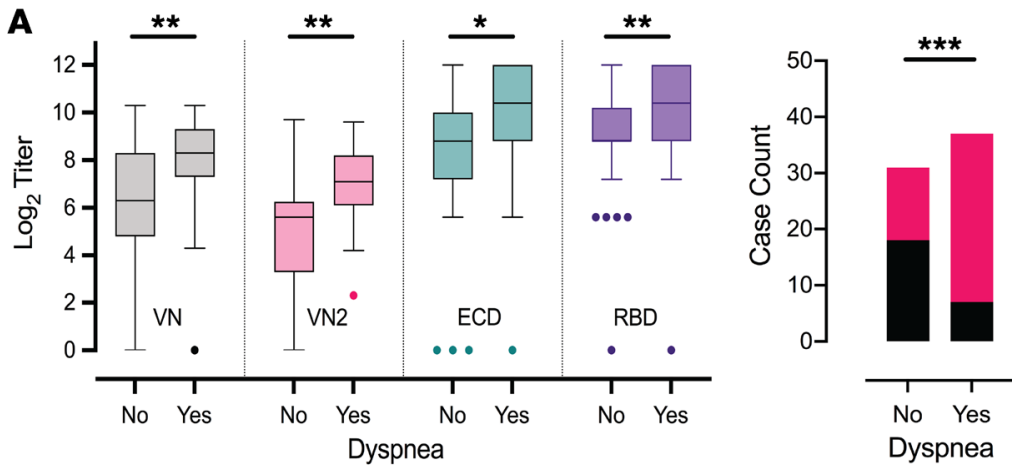

RR $3.1(95 \%$ Cl: $1.5-6.4)$
OR $5.9(95 \%$ Cl: $1.9-16.7)$
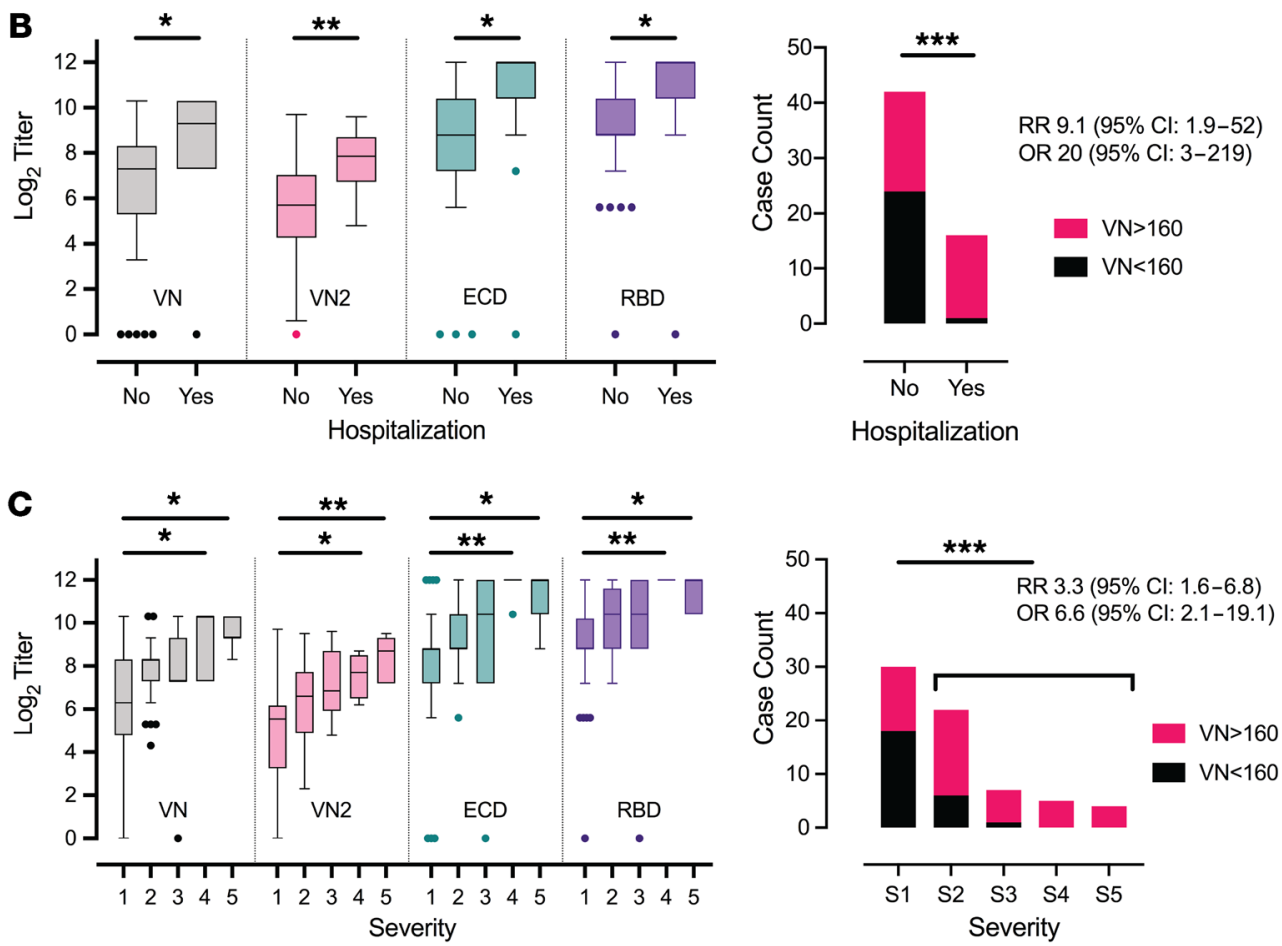

Figure 3. Distributions of VN, VN2, anti-ECD, and anti-RBD titers based on convalescent plasma donor self-reported clinical characteristics. Box plots of VN, VN2, anti-ECD and anti RBD titers by (A) dyspnea, (B) hospitalization, and (C) disease severity (1, low severity; 5, high severity) at initial plasma donation from the 68 individual donors. The median, minimum, maximum, first and third quartile, and extreme values are reported. Case counts of donors above and below the $\mathrm{VN} \geq 160$ threshold were stratified by whether they self-reported (A) occurrence of dyspnea during symptomatic phase of disease, (B) hospitalization, and (C) disease severity. Pairwise $t$ test $\left({ }^{*} P<0.05,{ }^{* *} P<0.01\right.$, $\left.{ }^{* *} P<0.001\right), 0 R$, and relative risk (RR) with $\mathrm{Cl}$ are also reported.

Of particular note, approximately one-third of convalescent plasma donors in our study did not meet the FDA-recommended cut-off of 1:160 for VN titer. This finding is consistent with the $60 \%$ that did not meet the target neutralization threshold of 1:100 recently described in the Harvala study (28). However, the inability to directly compare titers between laboratories highlights an unmet need for the development of international standards to enable comparisons of SARS-CoV-2 serological assays between laboratories (27). An increasing number of patients with COVID-19 are being treated globally with convalescent plasma. For example, under an FDA-approved expanded access protocol, $>50,000$ transfusions have already occurred in the US alone (8). Inasmuch as convalescent donor plasma likely will continue to play an important role in treatment of patients with COVID-19 for the foreseeable future, as efforts are made to manufacture polyclonal hyperimmune immunoglobulin and neutralizing monoclonal antibodies, especially as indications of efficacy are published (29), we felt it necessary to determine if certain donor characteristics may associate with high VN titer. We found that antibody titers were associated with disease severity and hospitalization status. Among all COVID-19 symptoms and donor characteristics assessed, the presence of dyspnea was the best symptom to discriminate the presence of an adequate IgG antibody titer. Although the sample size is small, we found that, even for donors who donated plasma twice per week for up to 7 donations, there was no significant decrease in titers, as assessed by the IgG ELISAs and VN. We believe these data could inform efforts to recruit plasma donors for therapeutic purposes. The finding that increased COVID-19 disease severity is associated with a more robust humoral immune response is consistent with 
previous studies of patients with SARS and dengue hemorrhagic fever (30) but contrasts with a recent report analyzing patients with COVID-19 (31). It is possible that differences in antibody testing platforms account for the contrasting observations. The list of emergency-use authorized antibody testing platforms is rapidly expanding, and test performance, especially as it relates to VN, will be important to understand (32). Regardless, our findings are in agreement with a more recent report, which found that strong antivirus antibody responses were associated with male sex, older age, and hospitalization (33).

Analysis of the available genomes for the SARS-CoV-2 strain pairs infecting convalescent donors and recipients found few differences in the inferred amino acid sequences and no association among magnitude of humoral immunity, disease severity, or infecting strain genotype. Because our sample size is small, more work is required in this area.

Several important matters remain unanswered with respect to anti-S protein IgG antibodies. First, although many believe, and some experimental animal infection data support (34), that antibodies directed against $\mathrm{S}$ protein confer protection from SARS$\mathrm{CoV}-2$ infection or reinfection, this remains unproven in humans. Second, although our data and work by others show a strong relationship between anti-S protein IgG titers and in vitro $\mathrm{VN}$, it will be important to determine if IgG antibody titer against this protein is a significant correlate of protective immunity in humans. This is an especially important topic given the massive efforts globally on using $S$ protein as a vaccine.

Our study has several limitations. The study was retrospective, only IgG titers were analyzed, and all VN studies were conducted in vitro. Plasma from the convalescent donors was used for VN assays, whereas serum samples were used for ELISAs. As such, the findings may not be entirely applicable to all antibody testing platforms or other sample types. Given the timing of the study relative to the pandemic curve in the Houston metropolitan region, donors were, at most, 53 days after symptom onset. Additional studies with donors that are later in their convalescence are needed. The sample size was limited by the number of donors recruited for plasma collection, and additional studies with larger sample sizes are needed. However, the data represent the most extensive assessment of the correlation between independent live VN and ELISAs for anti-SARS-CoV-2 antibodies in convalescent plasma donors to date.

Conclusions. Taken together, the data clearly show that antiRBD and anti-ECD IgG titers serve as important surrogates for in vitro VN activity. A substantial fraction of convalescent plasma donors may have VN titers below the FDA-recommended cut-off of $\geq 1: 160$. Dyspnea, hospitalization, and higher disease severity were associated with higher VN titer. Importantly, a small percentage of asymptomatic individuals have virus-neutralizing antibodies, including some with a titer of $\geq 1: 160$. In the aggregate, it is reasonable to think that our findings provide impetus for widespread implementation of anti-RBD and antiECD IgG antibody titer testing programs. The resulting data could be useful in several settings, including, but not limited to, identification of plasma donors for therapeutic uses (e.g., convalescent plasma transfusion and/or source plasma for fractionation in the manufacture of hyperimmune globulin) $(7,13)$, assessment of recipients of candidate vaccines, assessment of recipients of passive immune therapies, assessment of previously infected individuals, and identification of asymptomatic individuals with antibodies against SARS-CoV-2.

\section{Methods}

Convalescent plasma donors. Convalescent plasma was obtained by apheresis using the Trima Accel automated blood collection system (Terumo BCT) and processed by standard blood banking protocols. FDA recommendations for COVID-19 convalescent plasma donor collection were followed (7). Each donor had laboratory-confirmed SARS-CoV-2 infection based on a positive RT-PCR test. All plasma was donated by recovered and healthy patients with COVID-19 who had been asymptomatic for more than 14 days. Donors were between 18 and 65 years old. All donors tested negative for SARS-CoV- 2 at the time of plasmapheresis. If eligible according to standard blood donor criteria, donors were enrolled in a frequent plasmapheresis program. Donors were documented to be negative for anti-HLA antibodies, hepatitis B and C, HIV, human T-lymphotropic virus I/II, Chagas disease, West Nile virus, Zika virus, and syphilis per standard blood banking practices. Disease symptoms (fever, chills, productive or nonproductive cough, dyspnea, fatigue, myalgias, headache, runny nose, sore throat, nausea, vomiting, diarrhea, abdominal discomfort, loss of smell or taste, and other), disease severity, hospitalization requirement, and hospitalization course were assessed for each donor. A severity score was assigned as follows: 0 , asymptomatic; 1 , mild disease without dyspnea; 2 , moderate disease with dyspnea that did not require hospitalization; 3 , moderate disease with dyspnea that required hospitalization; 4 , severe disease that required supplemental oxygen; and 5, critical disease that required intensive care unit admission and/or intubation/mechanical ventilation. An aliquot of convalescent plasma product was used for virus microneutralization assays.

Asymptomatic donors and VN titers. Samples from asymptomatic individuals were obtained from volunteers screened through a Houston Methodist IRB-approved community surveillance protocol (22). Analysis of 2814 asymptomatic adults found that 73 (2.5\%) had an anti-ECD of $\geq 1: 50$. These were analyzed for anti-RBD and VN titer (Supplemental Table 1).

Specimens from SARS-CoV-2 naive donors. Ten naive human plasma specimens (negative controls) were obtained from samples biobanked in Houston well before SARS-CoV-2 was described in China, the US, or elsewhere.

$R T-P C R$ resting for SARS-CoV-2 infection. Symptomatic patients with a high degree of suspicion for COVID-19 disease were tested at the Molecular Diagnostics Laboratory at Houston Methodist Hospital using an assay filed for under Emergency Use Authorization from the US FDA (33). The assay follows the protocol published by the World Health Organization (35) and uses a 7500 Fast Dx instrument (Applied Biosystems) and 7500 SDS software (Applied Biosystems). Testing was performed on nasopharyngeal or oropharyngeal swabs immersed in universal transport media, bronchoalveolar lavage fluid, or sputum treated with dithiothreitol.

SARS-CoV-2 ELISAs. Detailed ELISA methods have been recently described (36). The ELISA used to measure anti-S IgG antibodies in donor serum specimens was performed as follows. Briefly, ECD-purified recombinant protein used comprises amino acid residues 1-1208, and the RBD comprises amino acids 319-591 of SARS-CoV-2 S pro- 
tein (GenBank MN908947). Microtiter plates were coated with either purified recombinant SARS-CoV-2 ECD or RBD. Human monoclonal antibody CR3022 that targets the RBD of SARS-CoV (37) was used as a positive control. Negative serum control was included on each microtiter plate. Serial dilutions of serum were added, incubated for 1 hour, washed, incubated with goat anti-human IgG Fab horseradish peroxidase (MilliporeSigma A0293), and washed. ELISA substrate (1-step Ultra TMB, Thermo Fisher Scientific, 34028) was added, the plates were developed until the top dilution reached the saturation point, and the reaction was stopped with $\mathrm{H}_{2} \mathrm{SO}_{4}$. Plates were read at an absorbance of $450 \mathrm{~nm}$.

A similar ELISA was used to study anti-S ECD antibody titers in serum obtained from surveilled asymptomatic individuals. Recombinant proteins were produced as described above. All samples were tested with an initial screen assay, and IgG antibody titers were subsequently performed on positive samples. For the screening assay, patient serum samples and negative control samples were diluted 1:50 in PBS containing $2 \%$ nonfat milk before addition to the plate. Patient sera that were identified as positive by the screening assay were subsequently titered by 1:3 serial dilutions in PBS-M to create 1:50, 1:150, $1: 450,1: 1350$, and 1:4050 final dilutions. Titer was defined as the last dilution showing an optical density greater than average negative control plus 3 standard deviations.

SARS-CoV-2 microneutralization assay (VN). The ability of plasma samples to neutralize SARS-CoV-2 host-cell infection was determined with a traditional VN assay using SARS-CoV-2 strain USA-WA1/2020 (NR-52281-BEI resources), as previously described for SARS-CoV (17). The assay was performed in triplicate, and a series of 8 two-fold serial dilutions of the plasma or serum were assessed. Briefly, 100 tissue culture infective dose $50\left(\mathrm{TCID}_{50}\right)$ units of SARS-CoV-2 was added to 2 -fold dilutions of heat-inactivated serum or plasma, and incubated for 1 hour at $37^{\circ} \mathrm{C}$. The virus and plasma mixture was added to Vero E6 cells (ATCC CRL-1586) grown in a 96-well microtiter plate and incubated for 3 days, after which the host cells were treated for 1 hour with crystal violet-formaldehyde stain (0.013\% crystal violet, $2.5 \%$ ethanol, and $10 \%$ formaldehyde in $0.01 \mathrm{M} \mathrm{PBS})$. The endpoint of the microneutralization assay was designated as the highest plasma dilution, at which all 3, or 2 of 3 , wells are not protected from virus infection, as assessed by visual examination.

SARS-CoV-2 microneutralization assay 2. A second SARS-CoV-2 microneutralization assay (VN2) was adapted from an assay used to study Ebola virus (38). This assay also used SARS-CoV-2 strain WA1. Plasma specimens were heat inactivated in a $56^{\circ} \mathrm{C}$ water bath for 30 minutes to inactivate complement. Heat-inactivated plasma specimens were diluted 1:10 in cell culture media (MEM; Corning, 10-010) containing 2\% FBS (GE Healthcare Hyclone), and 3-log dilutions were performed in duplicate. Plasma from naive and SARS-CoV-2 convalescent individuals was used as a negative and positive control, respectively. Diluted plasma was mixed with the SARS-CoV-2 WA1 strain, incubated at $37^{\circ} \mathrm{C}$ for 1 hour, and then added to Vero E6 cells at a target MOI of 0.4. Unbound virus was removed after 1-hour incubation at $37^{\circ} \mathrm{C}$, and cells were washed once in Dulbecco's Phosphate-Buffered Saline without calcium and magnesium (DPBS, MilliporeSigma) and culture media (MEM + 5\% FBS + 1\% Penicillin-Streptomycin) (Gibco Thermo Fisher Scientific, 15140122) was added. Cells were fixed 24 hours after infection, washed 3 times with DPBS, permeabilized with $1 \%$ Triton X-100 (Bio-Rad), and blocked with Cell Staining Buf- fer (BioLegend). The number of infected cells was determined using SARS-CoV-nucleocapsid-specific monoclonal antibody (Sino Biological, 401430-R001) and goat anti-mouse cross-adsorbed IgG (H\&L) Alexa Fluor 488 fluorescently labeled secondary antibody (Invitrogen Thermo Fisher Scientific, A-11001) and NucBlue Live ReadyProbes Reagent (Hoechst 33342) (Invitrogen Thermo Fisher Scientific). The percentage of infected cells was determined with an Operetta high-content imaging system (PerkinElmer) and Harmonia software (39). Percentage neutralization for each plasma sample at each dilution was determined relative to untreated, virus-only control wells.

SARS-CoV-2 genome sequencing and analysis and clade assignment. Libraries for whole-virus genome sequencing were prepared according to version 1 or 3 of the ARTIC nCoV-2019 sequencing protocol (40). Long reads were generated with the LSK-109 sequencing kit, 24 native barcodes (NBD104 and NBD114 kits), and a GridION instrument (Oxford Nanopore). Short reads were generated with the NexteraXT kit and a MiSeq or NextSeq 550 instrument (Illumina). Whole-genome alignments of consensus virus genome sequence generated from the ARTIC nCoV-2019 bioinformatics pipeline were trimmed to the start of orf1ab and the end of orf10 and used to generate a phylogenetic tree using RAxML (https://cme.h-its.org/exelixis/web/software/ raxml/index.html). Trees were visualized and annotated with CLC Genomics Workbench v20 (QIAGEN). SARS-CoV-2 clade assignment was based on procedures described elsewhere (41).

Statistics. To assess the correlation among VNs, anti-RBD, and anti-ECD ELISA titer data, pairwise Pearson correlations were performed using the entire data set (i.e., individuals with single and repeated measurements) using the psych package in $\mathrm{R}$ and a scatter plot of matrices, bivariate scatter plots, histograms, and the Pearson correlation determined with the pairs.panels function. To identify the prevalence of donors with VN titers of $\geq 1: 160$, the frequency distribution of these cases by titer classes critical for RBD, ECD, and VN2 was quantified. The generalized liner model, using the first plasma donation data only, was performed between the same variables, as a response, and each of the following predictor factors: dyspnea (yes, no), disease severity ( 5 classes, as described above), hospitalization (yes, no), sex (male, female), and age combined into 5 age groups ( $\leq 30,31-40,41-50,51-60$, and $>60$ years). For variables with more than 2 factors, a post hoc 2-tailed $t$ test (with Bonferroni's correction) was used to identify significant pairwise differences. A linear mixed-effect model was used to analyze the relationship among VNs, anti-RBD, and anti-ECD protein titers, as responses, and days since symptoms, as numerical predictors. Here, we used the whole data set and included the individual ID as the random factor to consider multiple sampling. A similar analysis was used for duration of symptoms but using generalized liner model and selecting only the cases at the first visit. Analyses were performed using $\log _{2}$-transformed numeric data and the R statistical computing platform (42). $P$ values of less than 0.05 were considered significant.

Study approval. Convalescent plasma was obtained and processed by standard blood banking protocols under Houston Methodist human subjects protocol PRO00025121. FDA recommendations for COVID-19 convalescent plasma donor collection were followed (7). All donors provided written informed consent. Studies were conducted with the approval of the Houston Methodist Research Institute ethics review board and with informed patient or legally authorized representative consent when applicable. 


\section{Author contributions}

$\mathrm{ES}, \mathrm{VK}$, and JMM conceived the project. ES, SVK, PAC, TE, XY, PZ, ZJ, SWL, RJO, J. Chen, BC, DT, JL, JG, J. Cardona, GI, RN, IB, DG, RMR, AG, SS, IP, IMC, NMJ, LP, KH, AH, JMD, and VK acquired data. VK, SVK, IMC, JMM, ES, and PAC analyzed data. ES, VK, and JMM wrote the manuscript. ES, SVK, IMC, and VK prepared figures. CL, PJH, and DWB provided scholarly advice. All authors revised the manuscript and gave final approval for publication.

\section{Acknowledgments}

We are deeply indebted to all of our volunteer plasma donors for their time, their gift, and their solidarity. We thank Katharine G. Dlouhy, Curt Hampton, and their team of coordinators and recruiters for outstanding efforts; Monisha Dey, Cheryl ChavezEast, John Rogers, Ahmed Shehabeldin, David Joseph, Guy Williams, Karen Thomas, and Curt Hampton, who were instrumental in efficiently managing the donor center; Jessica Thomas and Zejuan Li, Erika Walker, the very talented and dedicated molecular technologists, and the many labor pool volunteers in the Molecular Diagnostics Laboratory for their dedication to patient care; the many donor center and blood bank phlebotomists and technologists for their dedication to donor and blood safety; Sasha Pejerrey, Adrienne Winston, and Heather McConnell for editorial assistance; Brandi Robinson, Harrold Cano, and Cory Romero for technical assistance; Claude Moussa, Heather Patton, and the many members of the laboratory information technology team for rapidly implementing the necessary electronic workflows; Pamela McShane, Dilzi Mody, and the many members of the biorepository team for their meticulous management of patient samples; and Christina Talley, Susan Miller and Mary Clancy for consistent, thorough, and outstanding advice. We express our gratitude to Manuel Hinojosa and Mark Vassallo for their extensive efforts to rapidly procure resources and Roberta Schwartz for her efforts in implementing screening of asymptomatic individuals. We are indebted to Marc Boom and Dirk Sostman for their support and to many Houston citizens and businesses for their tremendous philanthropic support of this ongoing project, including, but not limited to, anonymous, Ann and John Bookout III, Carolyn and John Bookout, Ting Tsung and Wei Fong Chao Foundation, Ann and Leslie Doggett, Freeport LNG, the Hearst Foundations, Jerold B. Katz Foundation, C. James and Carole Walter Looke, Diane and David Modesett, the Sherman Foundation, Paula and Joseph C. "Rusty" Walter III, and Aramco Americas. Jason S. McLellan (University of Texas at Austin) provided the monoclonal antibody CR3022 and the S protein expression vectors, and we thank the members of the Center for Systems and Synthetic Biology at the University of Texas at Austin for technical assistance. We thank Terumo BCT for continuously and rapidly supplying blood collection devices and supplies and Victoria Cavener, Meera Surendran Nair, and Team COVID-19 serology at Penn State for their timely and technical assistance and logistical support. We thank Nancy Jenkins and Neal Copeland for critical reading of the manuscript. This study was supported by the Fondren Foundation and Houston Methodist Hospital and Research Institute (to JMM). This research has been funded in whole or part with federal funds under a contract from the National Institute of Allergy and Infectious Diseases, NIH contract 75N93019C00050 (to JL and GI). A portion of this work was funded through Cooperative Agreement W911NF-12-1-0390 by the Army Research Office (to JG). We acknowledge seed funding from the Huck Institutes of the Life Sciences for the studies at Penn State (to SVK), together with the Huck Distinguished Chair in Global Health award (to VK). Funding was also provided through the CARES Act, with programmatic oversight from the Military Infectious Diseases Research Program (to JMD). Opinions, discussions, conclusions, interpretations, and recommendations are those of the authors and are not necessarily endorsed by the US Army. The mention of trade names or commercial products does not constitute endorsement or recommendation for use by the Department of the Army or the Department of Defense. The graphical abstract was created using BioRender.

Address correspondence to: James M. Musser, Houston Methodist Hospital, 6565 Fannin St., B490, Houston, Texas 77030, USA. Phone: 713.441.5890; Email: jmmusser@houstonmethodist.org.
1. Wu F, et al. Author correction: a new coronavirus associated with human respiratory disease in China. Nature. 2020;580(7803):E7.

2. Centers for Disease Control and Prevention. United States COVID-19 Cases and Deaths by State. CDC COVID Data Tracker. https://www. cdc.gov/coronavirus/2019-ncov/cases-updates/ cases-in-us.html. Accessed June 1, 2020.

3. Beigel JH, et al. Remdesivir for the treatment of Covid-19 - preliminary report. $N$ Engl J Med. 2020;NEJMoa2007764.

4. Imai M, et al. Syrian hamsters as a small animal model for SARS-CoV-2 infection and countermeasure development. Proc Natl Acad Sci U S A. 2020;117(28):16587-16595.

5. Joyner MJ, et al. Evidence favouring the efficacy of convalescent plasma for COVID-19 therapy [preprint]. https://doi.org/10.1101/2020.07.29.2 0162917. Posted on medRxiv August 28, 2020.

6. National COVID-19 Convalescent Plasma Project. Component 3: Clinical Trials. https://ccpp19. org/healthcare_providers/component_3/index. html. Accessed June 1, 2020.

7. US Food \& Drug Administration. Recommendations for Investigational COVID-19 Convalescent Plasma. https://www.fda.gov/ vaccines-blood-biologics/investigational-newdrug-ind-or-device-exemption-ide-process-cber/ recommendations-investigational-covid-19convalescent-plasma. Accessed June 22, 2020. 8. COVID-19 Expanded Access Program. https:// www.uscovidplasma.org/. Accessed June 22, 2020.

9. Huang AT, et al. A systematic review of antibody mediated immunity to coronaviruses: antibody kinetics, correlates of protection, and association of antibody responses with severity of disease [preprint]. https://doi.org/10.1101/2020.04.14.2 0065771. Posted on medRxiv April 17, 2020.

10. Brouwer PJM, et al. Potent neutralizing antibodies from COVID-19 patients define multiple targets of vulnerability. Science. 2020;369(6504):643-650.

11. Premkumar L,et al. The RBD of the spike protein of SARS-group coronaviruses is a highly specific target of SARS-CoV-2 antibodies but not other pathogenic human and animal coronavirus antibodies. https://doi.org/10.1101/2020.05.06.200 93377. Posted on medRxiv May 10, 2020.

12. Suthar MS, et al. Rapid generation of neutralizing antibody responses in COVID-19 patients. Cell Rep Med. 2020;1(3):100040.

13. Amanat F, et al. A serological assay to detect SARS-CoV-2 seroconversion in humans. Nat Med. 2020;26(7):1033-1036.

14. Long QX, et al. Antibody responses to SARSCoV-2 in patients with COVID-19. Nat Med. 2020;26(6):845-848.

15. Wang C, et al. A human monoclonal antibody blocking SARS-CoV-2 infection. Nat Commun. 2020;11(1):2251.

16. Wu Y, et al. A noncompeting pair of human neutralizing antibodies block COVID-19 virus binding to its receptor ACE2. Science. 2020;368(6496):1274-1278.

17. Sui J, et al. Potent neutralization of severe acute respiratory syndrome (SARS) coronavirus by a human $\mathrm{mAb}$ to $\mathrm{S} 1$ protein that blocks 
receptor association. Proc Natl Acad Sci US A. 2004;101(8):2536-2541.

18. Prabakaran P, et al. Structure of severe acute respiratory syndrome coronavirus receptor-binding domain complexed with neutralizing antibody. J Biol Chem. 2006;281(23):15829-15836.

19. Zhu Z, et al. Potent cross-reactive neutralization of SARS coronavirus isolates by human monoclonal antibodies. Proc Natl Acad Sci U S A. 2007;104(29):12123-12128.

20. Sui J, et al. Broadening of neutralization activity to directly block a dominant antibody-driven SARS-coronavirus evolution pathway. PLoS Pathog. 2008;4(11):e1000197.

21. Corti D, et al. Prophylactic and postexposure efficacy of a potent human monoclonal antibody against MERS coronavirus. Proc Natl Acad Sci US A. 2015;112(33):10473-10478.

22. Vahidyet F, et al. Prevalence of SARS-CoV-2 infection among asymptomatic healthcare workers in greater Houston: a cross-sectional analysis of surveillance data from a large healthcare system [preprint]. https://doi.org/10.1101/2020.05 21.20107581. Posted on medRxiv May 25, 2020.

23. Berry JD, et al. Neutralizing epitopes of the SARSCoV S-protein cluster independent of repertoire, antigen structure or mAb technology. MAbs. 2010;2(1):53-66.

24. Lan J, et al. Structure of the SARS-CoV-2 spike receptor-binding domain bound to the ACE2 receptor. Nature. 2020;581(7807):215-220.

25. Walls AC, Park YJ, Tortorici MA, Wall A, McGuire AT, Veesler D. Structure, function, and antigenicity of the SARS-CoV-2 spike glycoprotein. Cell. 2020;181(2):281-292.e6.

26. Li L, et al. Effect of convalescent plasma therapy on time to clinical improvement in patients with severe life-threatening COVID-19: a randomized clinical trial.

JAMA. 2020;324(5):460-470.

27. Oguntuyo KY, et al. Quantifying absolute neutralization titers against SARS-CoV-2 by a standardized virus neutralization assay allows for cross-cohort comparisons of COVID-19 sera [preprint]. https://doi.org/10.1101/2020.08.13.2 0157222. Posted on medRxiv August 15, 2020.

28. Harvala $\mathrm{H}$, et al. Convalescent plasma therapy for the treatment of patients with COVID-19: assessment of methods available for antibody detection and their correlation with neutralising antibody levels [preprint]. https://doi.org/10.1101/2020.05. 20.20091694. Posted on medRxiv May 26, 2020.

29. Joyner MJ, et al. Effect of convalescent plasma on mortality among hospitalized patients with COVID-19: initial three-month experience. https://doi.org/10.1101/2020.08.12.20169359. Posted on medRxiv August 12, 2020.

30. Lee N, et al. Anti-SARS-CoV IgG response in relation to disease severity of severe acute respiratory syndrome. J Clin Virol. 2006;35(2):179-184.

31. Phipps WS, et al. SARS-CoV-2 antibody responses do not predict COVID-19 disease severity. Am J Clin Pathol. 2020;154(4):459-465.

32. US Food \& Drug Administration. Emergency Use Authorizations for Medical Devices. https:// www.fda.gov/medical-devices/emergency-situations-medical-devices/emergency-use-authorizations. Accessed June 1, 2020.
33. Klein SL, et al. Sex, age, hospitalization drive antibody responses in a COVID-19 convalescent plasma donor population. JClin Invest. 2020;130(11):6141-6150.

34. Yu J, et al. DNA vaccine protection against SARS-CoV-2 in rhesus macaques. Science. 2020;369(6505):806-811.

35. Okba NMA, et al. Severe acute respiratory syndrome coronavirus 2-specific antibody responses in coronavirus disease 2019 patients. Emerg Infect Dis. 2020;26(7):1478-1488.

36. Salazar E, et al. Treatment of COVID-19 patients with convalescent plasma. Am J Pathol. 2020;190(8):1680-1690.

37. ter Meulen J, et al. Human monoclonal antibody combination against SARS coronavirus: synergy and coverage of escape mutants. PLoS Med. 2006;3(7):e237.

38. Dean CL, et al. Characterization of Ebola convalescent plasma donor immune response and psoralen treated plasma in the United States. Transfusion. 2020;60(5):1024-1031.

39. Wec AZ, et al. Antibodies from a human survivor define sites of vulnerability for broad protection against ebolaviruses. Cell.2017;169(5):878-890.e15.

40. SARS-CoV-2. https://artic.network/ncov-2019. Accessed June 1, 2020.

41. Long SW, et al. Molecular architecture of early dissemination and evolution of the SARS-CoV-2 virus in metropolitan Houston, Texas [preprint]. https://doi.org/10.1101/2020.05.01.072652. Posted on bioRxiv May 03, 2020.

42. The R Project for Statistical Computing. https:// www.r-project.org/. Accessed August 8, 2020. 